\title{
MODELAGEM DE DISTRIBUIÇÕES DE TAMANHO DE PARTÍCULAS, USANDO FUNÇÃO LOGNORMAL MULTIMODAL EM PROCESSOS DE POLIMERIZAÇÃO EM MEIO HETEROGÊNEO
}

\author{
J. C. FERRARI ${ }^{1}$, C. SAYER ${ }^{1}$ e P.H.H. ARAÚJO ${ }^{1}$, F. de CASTILHOS ${ }^{2}$ \\ ${ }^{1}$ Universidade Federal de Santa Catarina, Departamento de Engenharia Química e Engenharia de \\ Alimentos \\ ${ }^{2}$ Universidade Federal de Santa Maria, Departamento de Engenharia Química \\ jarbasferrari@yahoo.com.br
}

\begin{abstract}
RESUMO - O entendimento da dinâmica da distribuição do tamanho de partícula durante o processo de polimerização é muito importante para a qualidade final do látex polimérico. Por conta disso, a modelagem da distribuição do tamanho de partícula em reatores de polimerização assume seu espaço na literatura científica, através das equações de balanço populacional que, no entanto, apresenta limitação quanto à determinação de parâmetros cinéticos e esforço computacional. Este trabalho tem por objetivo propor a utilização de uma função densidade de probabilidade lognormal para descrever as curvas de distribuição de tamanho de partícula em processos de polimerização. A estimação de parâmetros do modelo proposto é favorecida pela aplicação do algoritmo de otimização global Particle Swarm Optimization. A avaliação estatística dos resultados indica uma forte correlação entre distribuições experimentais e calculadas.
\end{abstract}

\section{INTRODUÇÃO}

A distribuição do tamanho de partícula (DTP) é uma das características mais importantes do látex/resina polimérico, de forma que propriedades como a viscosidade, o conteúdo máximo de sólidos, adesão e tempo de secagem dependem do perfil dessa distribuição (Vale e McKenna, 2005). Nesse contexto, a determinação das DTPs assume grande importância para qualquer proposta de tenha como objetivo o monitoramento e o diagnóstico das condições ótimas de operação de um sistema reacional de polimerização em meio heterogêneo.

Apesar dos avanços recentes nas metodologias de determinação experimental destas distribuições, ainda persistem dificuldades relacionadas ao preparo prévio da amostra podendo apresentar certa complexidade e excesso de tempo de análise, além da falta de reprodutibilidade no caso de distribuições polidispersas e ou com partículas muito pequenas. Uma alternativa é a modelagem fenomenológica fazendo uso das equações de balanço populacional (Kiparissides et al. 2002; Araújo et al. 2001; Coen et al. 2004), sendo que estes modelos permitem uma estrutura que 


\section{9 a 22 de outubro de 2014 \\ Florianópolis/SC}

pode relacionar a DTP à cinética de reação, à nucleação e crescimento das partículas, bem como a coagulação. No entanto, de acordo com Vale e McKenna (2005), mesmo quando superadas as dificuldades relativas à modelagem da coagulação, as equações de balanço populacional são difíceis de resolver se as mesmas incluírem modelos cinéticos e ou hidrodinâmicos completos.

Caminhos alternativos na avaliação do comportamento das DTPs podem ser a utilização de ferramentas de fluidodinâmica computacional (Alexopoulos et al. 2002) ou a inferência destas distribuições por meio de redes neurais (Gugliotta, et al. 2009). Em ambos os casos, uma função densidade de probabilidade pode ser usada para descrever as distribuições de tamanho de partícula.

Diante do exposto, este trabalho tem por objetivo, propor a aplicação de uma função lognormal multimodal para descrever o comportamento de distribuições de tamanho de partícula em processos de polimerização em meio heterogêneo, com parâmetros estimados com o auxílio do algoritmo de otimização Particle Swarm Optimization. A referida função apresenta inúmeras aplicações (Ryan, 2009; Rausand e Hoyland, 2004; Maring et al. 2003; Yuan et al. 2011; Hwang e Choi, 2006) mostrando-se eficiente e flexível em descrever distribuições, sejam elas unimodais ou multimodais, largas ou estreitas.

\section{MATERIAIS E MÉTODOS}

\subsection{Distribuições de tamanho de partícula experimentais}

No intuito de imprimir credibilidade à proposta de modelagem da DTP usando a função lognormal multimodal foram realizadas estimações usando dados de distribuições de tamanho de partícula experimentais de reações de polimerização em emulsão realizadas por Araújo (1999), apresentando como características principais a assimetria à direita das distribuições no início da nucleação, causado pela pré-alimentação do reator com excesso de surfactante e os inúmeros casos de multimodalidades, ocasionados pelas sucessivas renucleações. Além destas, uma DTP relatada no trabalho de Mallikarjunan et al. (2010) foi investigada por apresentar um tipo diferente de reator. As DTPs de reações de polimerização em suspensão, realizadas por Jahanzad (2004) também foram modeladas a fim de investigar a capacidade de adaptação da estratégia a outra forma de polimerização em meio heterogêneo sem, no entanto, a observância de distribuições muito complexas. A Tabela 01 detalha os autores e os sistemas reacionais de polimerização de onde as DTPs investigadas foram extraídas.

Tabela 1 - Apresenta os autores e os sistemas reacionais de polimerização heterogêneas

\begin{tabular}{|c|c|c|c|}
\hline Distribuição & Tipo reator & Reações & Autores (s) \\
\hline DTP01, DTP02, & Loop contínuo & $\begin{array}{c}\text { Polimerização em emulsão de } \\
\text { vinil acetato } \text { e Veova } 10- \\
\text { Reações } \text { RL10/RL5/RL4/RL10 }\end{array}$ & Araújo (1999) \\
\hline DTP04 & $\begin{array}{c}\text { Tanque agitado } \\
\text { Semi-batelada }\end{array}$ & $\begin{array}{c}\text { Polimerização em emulsão de } \\
\text { vinil acetato e butil acrilato. }\end{array}$ & $\begin{array}{c}\text { Mallikarjunan } \text { et al. } \\
\text { (2010) }\end{array}$ \\
\hline
\end{tabular}




\begin{tabular}{|c|c|c|c|}
\hline DTP05, DTP06 & $\begin{array}{c}\text { Tanque agitado } \\
\text { Batelada }\end{array}$ & $\begin{array}{c}\text { Polimerização em Suspensão } \\
\text { de Metil Metacrilato }\end{array}$ & Jahanzad (2004) \\
\hline
\end{tabular}

\subsection{Estimação de parâmetros}

A distribuição lognormal é um modelo com dois parâmetros, cuja função densidade é expressa pela Equação 01 (Zender, 2010):

$$
f\left(x, \mu_{g}, \sigma_{g}\right)=\frac{1}{x \cdot \ln \left(\sigma_{g}\right) \sqrt{2 \pi}} \cdot \exp \left[-\frac{1}{2}\left(\frac{\ln (x)-\ln \left(\mu_{g}\right)}{\sigma_{g}}\right)^{2}\right], \text { para } x>0
$$

onde, $\mu_{g}$ e $\sigma_{g}$ representa a média geométrica e o desvio padrão geométrico da distribuição, respectivamente e $x$ é a variável independente.

No caso em que os dados estiverem agrupados em distribuições de frequências, os valores $x_{k}$ serão ponderados pelas respectivas frequências relativas $f_{k}$, com $k=1,2, \ldots, n$. Nesse caso, a média geométrica e o desvio padrão geométrico podem ser definidos pelas Equações 02 e 03, respectivamente (Klink et al. 2011):

$$
\begin{gathered}
\ln \mu_{g}=\frac{1}{N} \sum_{k=1}^{n} f_{k} \ln \left(x_{k}\right)=\ln \sqrt[N]{\prod_{k=1}^{n}\left(x_{k}\right)^{f_{k}}} \\
\ln \sigma_{g}=\sqrt{\frac{\sum_{k=1}^{n} f_{k} \cdot\left(\ln \left(x_{k}\right)-\ln \mu_{g}\right)^{2}}{N-1}}
\end{gathered}
$$

, com $N=\sum_{k=1}^{n} f_{k}$ sendo a frequência total absoluta.

No entanto, em situações reais, tais distribuições podem ser compostas por mais de uma moda, podendo ser bimodal, trimodal ou n-modal. Nestes casos, a função pode ser descrita por meio de uma soma ponderada de mais de uma função densidade de probabilidade lognormal unimodal (Zender, 2010). A Equação 04 descreve a função densidade de probabilidade lognormal multimodal utilizada neste trabalho:

$$
F L M\left(d, \mu_{g}^{i}, \sigma_{g}^{i}, w^{i}\right)=\sum_{i=1}^{n} \frac{w_{i}}{d \cdot \ln \sigma_{g}^{i} \sqrt{2 \pi}} \cdot \exp \left[-\frac{1}{2}\left(\frac{\ln (d)-\ln \left(\mu_{g}^{i}\right)}{\ln \sigma_{g}^{i}}\right)^{2}\right], \text { para } \mathrm{d}>0
$$


onde, $\operatorname{FLM}\left(d, \sigma_{g}^{i}, \mu_{g}^{i}, w^{i}\right)$ : Função Lognormal Multimodal; $d$ é o diâmetro das partículas; $n$ : número de modas e $w^{i}$ : fator peso da moda $i$ admitindo que $\sum_{i=1}^{n} w^{i}=1$. Ressaltando ainda que há três parâmetros a serem estimados em cada moda $\left[\ln \mu_{g}^{i}\right.$ : logaritmo natural da média geométrica da moda $i$; $\ln \sigma_{g}^{i}$ : logaritmo natural do desvio padrão geométrico da moda $i ; w^{i}$ : fator peso da moda $\left.i\right]$.

Diante das características das distribuições investigadas neste trabalho, optou-se por realizar modelagens fazendo uso de uma Função Lognormal Trimodal [FLT] e uma Função Lognormal Bimodal [FLB], comparando os resultados posteriormente.

A robustez do processo de estimação de parâmetros do modelo é garantida pelo Particle Swarm Optimization que usa sua estratégia inteligente para, de forma randômica, minimizar a função objetivo definida pela soma dos erros médios quadrados [SMSE], de acordo com a Equação 05:

$$
S M S E=\frac{1}{n} \sum_{k=1}^{n}\left(f_{\exp }^{k}-F L T\left(d_{k}\right)\right)^{2}
$$

onde, $f_{\exp }^{k}$ : valor experimental da frequência no ponto $k ; F L M\left(d_{k}\right)$ : frequência calculada pela função lognormal multimodal no ponto $k ; n$ : número de pontos experimentais.

O PSO tem inúmeras estratégias relatadas na literatura científica, sendo que nesse trabalho foi adotado a proposta por Jiao et al:, (2006), que considera a implementação do peso inércia dinâmico, [Equação 06]. Os parâmetros do algoritmo seguem as referências de Jiao et al-, (2006).

$$
w=w_{0} \cdot u^{-k}
$$

Como se trata de uma estratégia que realiza busca randômica, é necessário definir os limites superiores e inferiores dos parâmetros a serem ajustados. Sendo assim, os parâmetros e seus respectivos intervalos de busca são: $\ln \mu_{g}[3.2,6.6] ; \ln \sigma_{g}[0.01,0.6]$ e $w[0,1]$.

As regressões foram realizadas em triplicata, de forma que os valores relatados como parâmetros ótimos são a média aritmética dos parâmetros encontrados nas três estimações. São relatados também os desvios padrão de cada parâmetro, salientando que estes desvios representam uma variabilidade do algoritmo em estimar aquele parâmetro específico já que o mesmo executa busca randômica, não tendo relação com o intervalo de confiança do parâmetro ajustado. Ressalta-se ainda que, a implementação da estratégia de estimação de parâmetros ocorreu a partir de um homemade program Fortran 90 linguage.

A interpretação estatística dos resultados foi realizada por meio do cálculo do coeficiente de correlação [r] entre as frequências experimentais e frequências calculadas pelo modelo após o ajuste dos parâmetros. Além disso, o teste de aderência de Kolmogorov-Smirnov foi utilizado para comparar 
as distribuições de frequência acumulada experimental com as distribuições de frequência acumulada estimada pela função lognormal multimodal.

\section{RESULTADOS E DISCUSSÕES}

A comparação entre DTPs experimentais e modelados pela função lognormal multimodal proposta está apresentada na Figura 01. Os gráficos demonstram claramente que a função lognormal trimodal se ajusta mais perfeitamente as distribuições experimentais, o que era de se esperar pelo número excessivo de parâmetros. Resultados igualmente satisfatórios foram obtidos pela função lognormal bimodal, salientando que nesse caso o número de parâmetros estimados é significativamente menor. Por fim, pode-se observar que a FLB ignora a terceira moda em DTP 02B, visto que é a moda representa menos de $8 \%$ da distribuição, além disso, o modelo em questão só propicia a geração de duas modas.

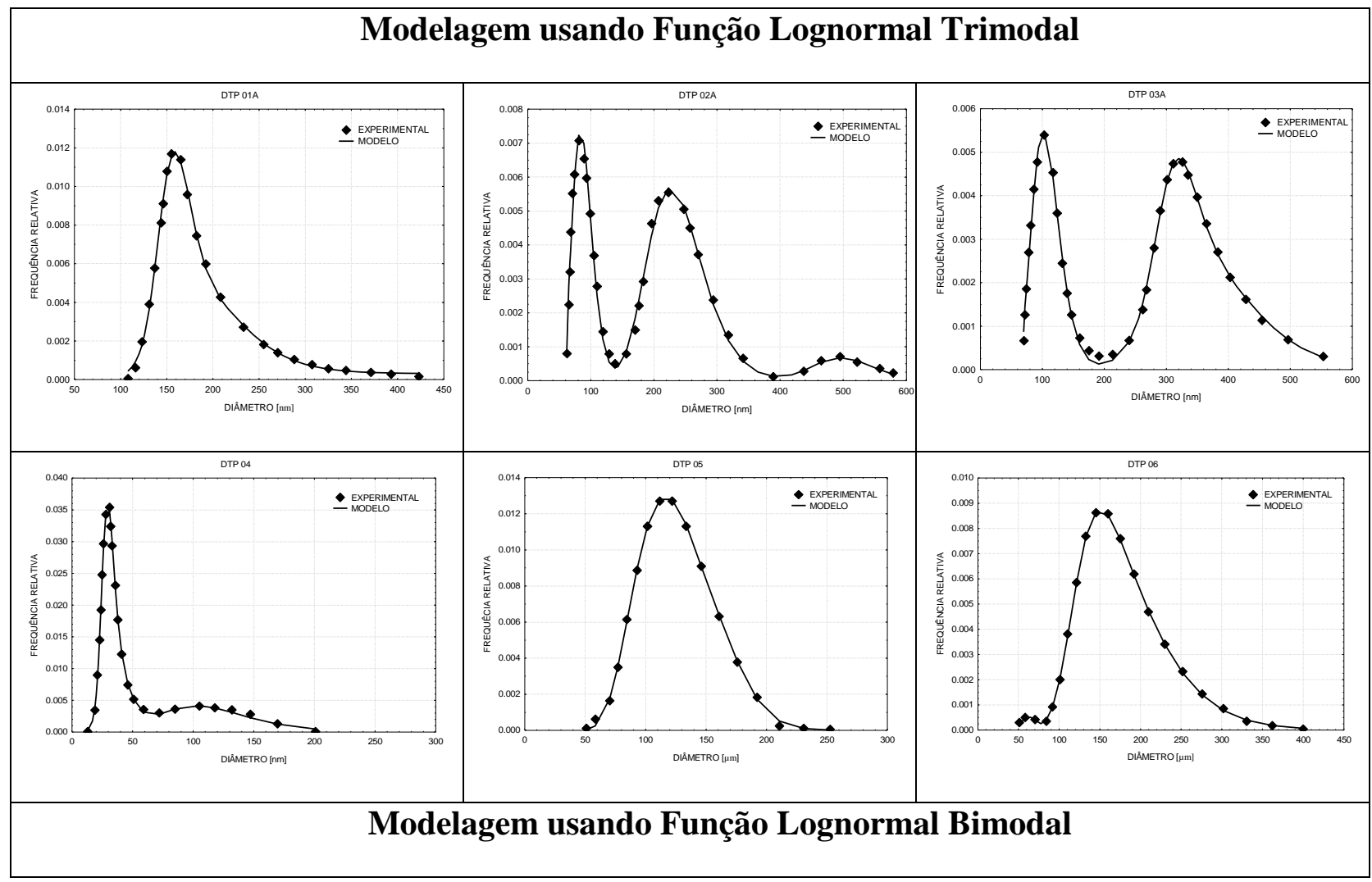




\section{1) LCOBEQ}

19 a 22 de outubro de 2014

Florianópolis/SC
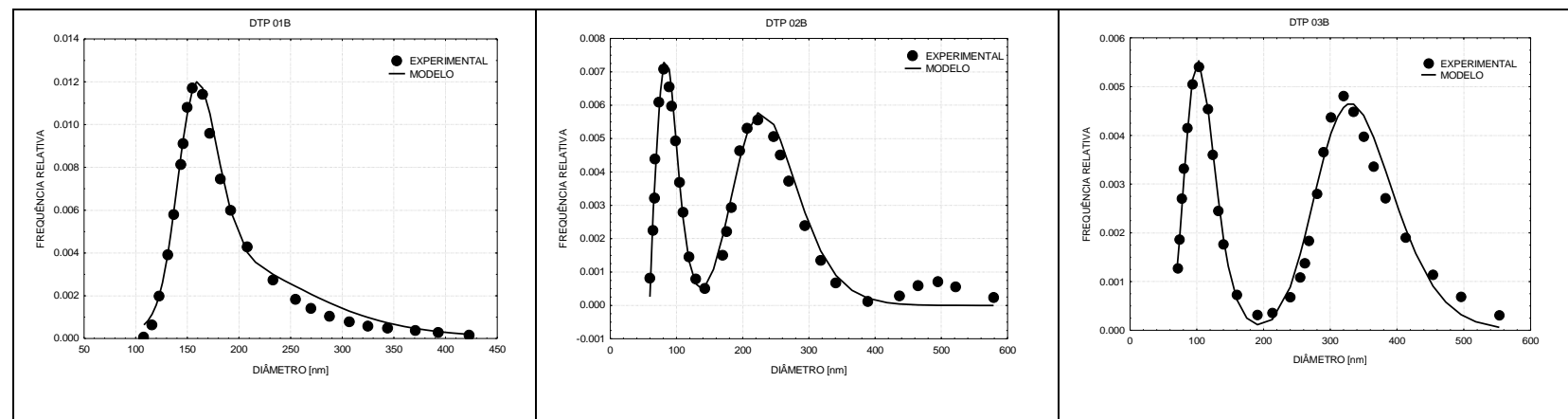

Figura 1 - Comparação entre as curvas de DTPs experimentais e calculadas pelos modelos propostos.

A Tabela 03 apresenta à média e o desvio padrão dos parâmetros ajustados para cada uma das DTPs investigadas e o valor da função objetivo [SMSE] ao final da regressão. A erro na estimação dos parâmetros foi quantificado a partir na análise da função objetivo, resultando em valores baixos em todos os casos investigados contribuindo para comprovar a robustez do PSO. Já os baixos desvios padrão dos parâmetros estimados, contribuíram para atestar a capacidade do algoritmo em reproduzir resultados consistentes.

Tabela 3 - Valores dos parâmetros ajustados para cada uma das DTPs investigadas

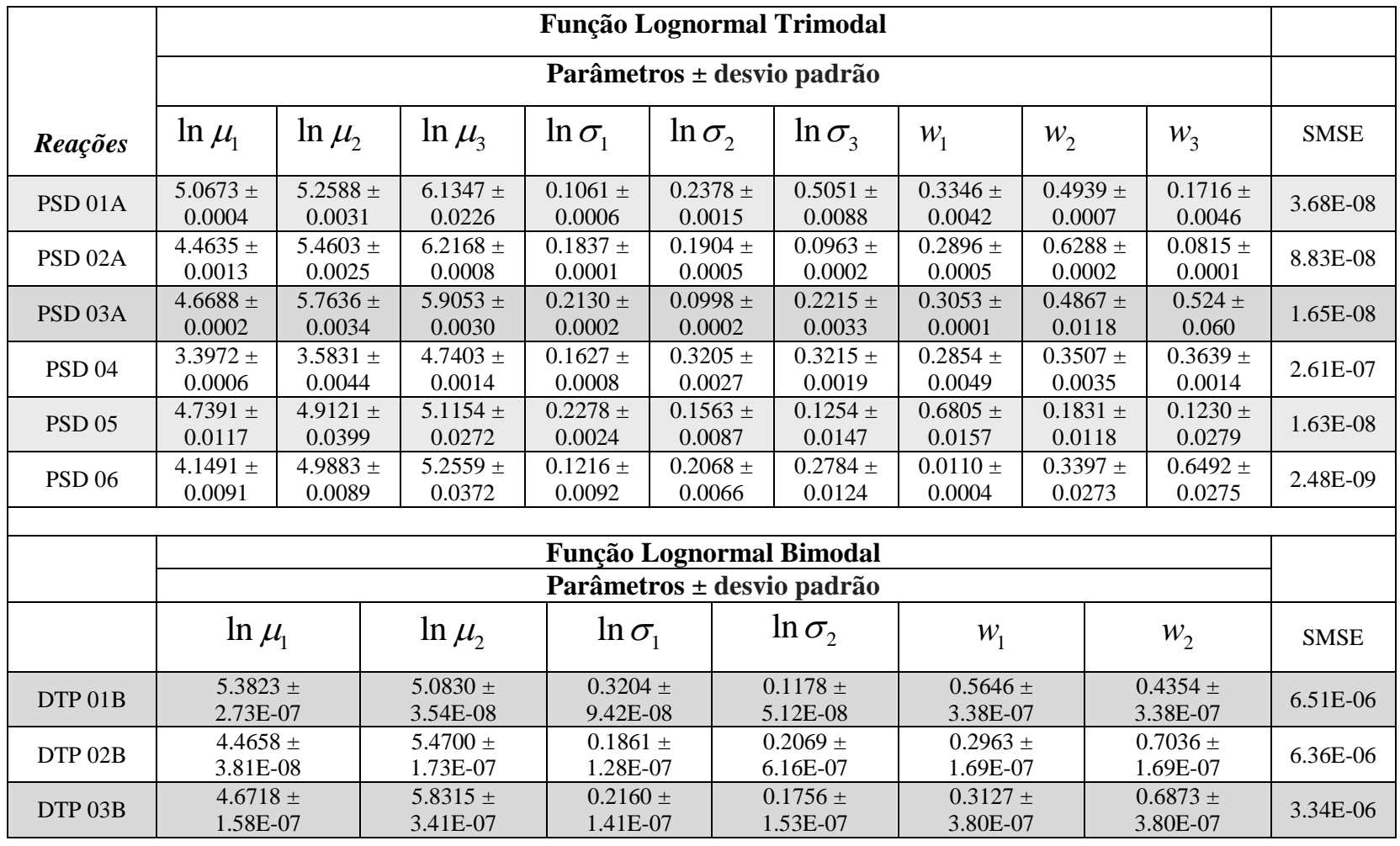

Os resultados apresentados na Tabela 3 demonstram que não há diferenças significativas nos 


\section{9 a 22 de outubro de 2014 \\ Florianópolis/SC}

resultados apresentados pela FLT e FLB. No entanto, a alta correlação entre os parâmetros da FLT é percebida quando avaliamos os valores dos desvios padrão apresentados, sendo muito menores para os parâmetros ajustados da FLB.

A avaliação do coeficiente de correlação [r] é coerente com os resultados apresentados na Figura 01, visto que $r \geq 0.98$ em todos os casos investigados estando, portanto as distribuições de frequência experimental e calculada fortemente correlacionadas. Com relação ao teste de aderência de Kolmogorov-Smirnov o maior valor para $\mathrm{d}_{\text {máx }}$ encontrado nos casos investigados foi $\mathrm{d}_{\text {máx }}=0.0147$. De acordo com os valores críticos estabelecidos pelo teste de Kolmogorov-Smirnov, deve-se admitir $\mathrm{d}_{\text {crítico }}=0.210$ para um nível de significância $\alpha=5 \%$ e para um número de pontos observados $\mathrm{n}=40$, aplicados nesse trabalho. Nesse caso, a hipótese de que os dados se referem a uma mesma distribuição deve ser aceita, acrescentando credibilidade à estimação de parâmetros realizada.

Por fim, observa-se que toda esta variabilidade presente nas distribuições de tamanho de partícula pode ser perfeitamente assimilada pela função lognormal bimodal com um custo computacional menor que no caso da função lognormal trimodal, não sendo observadas inconsistências na estratégia, desde que sejam observados os limites para o diâmetro da partícula que pode variar para diferentes mecanismos e sistemas, assim como considerar a utilização de frequências relativas normalizas pela área da curva da distribuição.

\section{CONCLUSÃO}

Uma função lognormal multimodal foi proposta para descrever distribuições de tamanho de partícula em reações de polimerização em meio heterogêneo, com parâmetros estimados pelo algoritmo de otimização global Particle Swarm Optimization. Foram modeladas distribuições com características distintas, sendo estas unimodais ou multimodais, largas ou estreitas, todas oriundas de reações de polimerização em emulsão ou suspensão.

A avaliação estatística dos resultados corrobora com a tese de que a função lognormal bimodal pode descrever, com sucesso, as mais diversas distribuições. Além disso, a precisão na estimação dos parâmetros, comprovada pelos baixos desvios padrão das triplicatas, contribui para atestar a capacidade do PSO em reproduzir resultados consistentes. Portanto, a utilização de uma função lognormal bimodal para descrever DTPs em processos de polimerização em meio heterogêneo constitui-se em uma alternativa coerente, observando que a utilização da função lognormal trimodal somente se justifica quando o sistema reacional apresentar uma dinâmica de nucleações sucessivas promovendo distribuições de partículas poliméricas polidispersas.

\section{REFERÊNCIAS}

ARAÚJO, P. H. H. Distribuição de Tamanho de Partícula em Sistemas Heterogêneos de Polimerização. Tese de Doutorado em Engenharia Química - Programa de Engenharia Química /COPPE - UFRJ, Rio de Janeiro, (1999).

ARAÚJO, P. H; DE LA CAL, J. C; ASÚA, J. M; PINTO, J. C. Modeling particle size distribution (PSD) in emulsion copolymerization reactions in a continuous loop reactor. Macromol Theory Simul. 

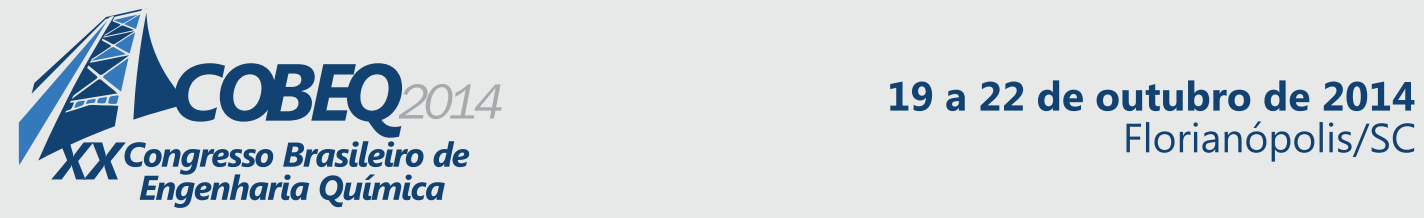

10:769-79 (2001).

COEN E. M; PEACH, S; MORRISON B. R; GILBERT R. G. First principles calculation of particle formation in emulsion polymerization: pseudo-bulk systems. Polymer; vol.45: 3595-608. (2004).

GUGLIOTTA, L. M; STEGMAYER, G. S; CLEMENTI, L. A; GONZALEZ, V. D. G; MINARI, R. J; LEIZA, J. R; VEGA, J. R. A Neural Network Model for Estimating the Particle Size Distribution of Dilute Latex from Multiangle Dynamic Light Scattering Measurements. Part. Syst. Charact.26, 4152, (2009).

HWANG, S.; CHOI, S. Use of a lognormal distribution model for estimating soil water retention curves from particle-size distribution data. Journal of Hydrology, vol. 323, Issues 1-4, 2006

JAHANZAD. F. Evolution of particle size distribution in suspension polymerization reactions. Submitted in partial fulfillment of the requirements for the award of the Doctor of Philosophy of the Loughborough University, 2004.

JIAO, B.; LIAN, Z.; GU, X. A dynamic inertia weight particle swarm optimization algorithm. Chaos Solitons \& Fractals. In press. 2006.

KIPARISSIDES, C., ACHILIAS, D.S., FRANTZIKINAKIS, C.E. The effect of oxygen on the kinetics and particle size distribution in vinylchloride emulsion polymerization. Ind. Eng. Chem. Res. 41, 3097-3109, (2002).

KLINK, I. M.; PHILLIPS, R. J.; DUNGAN, S. R. Effect of emulsion drop-size distribution upon coalescence in simple shear flow: A population balance study. Journal of Colloid and Interface Science 353 (2011).

LIMPERT, E.; STAHEL, W.; ABBT, M. Lognormal distribution across the sciences: Keys and Clues. BioScience, vol.51, Issue 5, 2001.

MALLIKARJUNAN, V; PUSHPAVANAM, S; IMMANUEL, C. D. Parameter estimation strategies in batch emulsion polymerization. Chemical Engineering Science, vol.65, pp.4967-4982. (2010).

MARING, H.; SAVOIE, D. L.; IZAGUIRRE, M. A.; CUSTALS, L.; REID, J. S. Mineral dust aerosol size distribution change during atmospheric transport, J. Geophys. Res., 108, 2003.

RAUSAND, M.; HOYLAND, A. System Reliability Theory: Models, Statistical Methods, and Applications. John Wiley \& Sons, 2004.

RYAN, T. P. Estatística Moderna para Engenharia. Tradução: Luiz Claudio de Queiros Faria. Elsevier. Rio de Janeiro, 2009.

VALE, H.M; McKENNA, T. F. Modeling particle size distribution in emulsion polymerization reactors. Progress in polymer Science. 30, 1019-1048, 2005.

YUAN ,Y.; YI, H.; SHUAI, Y.; LIU, B.; TAN, H. Inverse problem for aerosol particle size distribution using SPSO associated with multi-lognormal distribution model. Atmospheric Environment, vol. 45, 2011.

ZENDER, Charlie. Particle Size Distributions: Theory and Application to Aerosols, Clouds, and Soils. Online: http://dust.ess.uci.edu/facts, Updated: Mon 30th Aug, 2010. 